\section{Extensión universitaria, proyección social y su relación con la investigación y formación profesional en el marco del proceso de acreditación universitaria en la FII}

\section{RESUMEN}

Este trabajo, en síntesis, trata del ejercicio de buenas prácticas de gestión empresarial. El carácter sistémico de la administración, la alineación, la estrategia y habilidades en el sector de la minería metálica en el Perú, la influencia de la teoría de recursos y desarrollo de capacidades. También nos ocupamos de la cadena de valor, las ventajas competitivas, las estrategias y habilidades en la actividad minera metálica y estratos mineras. Desde el desarrollo de estos puntos, hemos creado como conclusiones que todos los estratos formales, legales 0 ilegales coexisten alguna manera, porque los legales pueden exportar toda la producción. Por otra parte, se requiere una estrategia, promoción, planificación y liderazgo profesional especializado en esta industria. Al mismo tiempo, es necesario que el apoyo del gobierno todo el esfuerzo y los centros de formación técnica y profesional en geología, minería, metalurgia, química y afines competencias definir y diseñar la formación de competencias para proporcionar el sector especializado del capital humano.

Palabras clave: alineamiento estratégico, capital humano, gestión, metalúrgica, mineria

UNIVERSITY EXTENSION, OUTREACH AND ITS RELATIONSHIP TO RESEARCH AND TRAINING IN THE CONTEXT OF THE UNIVERSITY ACCREDITATION IN THE FII

\section{ABSTRACT}

This work, in synthesis, is about the exercise of good corporate management practice. The systematic nature of administration, alignment, strategy and skills in the metal mining sector in peru, influence of the Resource and Capacity development theory. We also deal with the value chain, competitive advantages, strategies and skills in the metal mining activity and mining strata. From the development of these points, we set up as conclusions that all formal, legal or illegal strata coexist somehow because the legal ones can export all production. On the other hand, it is required a strategy, promotion, planning and specialized professional leadership in this industry. At the same time, it is required that the government support all this effort and the centers of technical and vocational training in geology, mining, metallurgy, chemistry and related competences define and design formation competence to provide the specialized human capital sector.

Keywords: human capital, management, metallurgical, mining, strategical alignment

\section{INTRODUCCIÓN}

Una de las funciones principales de la universidad peruana, junto a la formación profesional y la investigación, es su vinculación a la comunidad y a la sociedad que la alberga. Esto a través de la extensión universitaria y la proyección social.

El estudio comprendió la delimitación conceptual y operativa de los términos extensión universitaria y proyección social, en la facultad de Ingeniería Industrial de la UNMSM, y su relación con los procesos de formación profesional e investigación. En tal sentido, se enfatiza la necesidad de asumir y desarrollar una concepción integral de la extensión universitaria.

En las últimas décadas se ha puesto de relieve la gestión del conocimiento como el factor clave para la generación de ventajas competitivas. En tal sentido, la universidad, que desde su misión tiene como propósito la generación, difusión y transferencia de conocimiento a la sociedad, tiene un rol protagónico. Esto implica la necesidad de enfatizar la importancia de la función de extensión universitaria, por ser esta la que se encarga directamente de la interacción con el entorno.

En la Declaración de la Unesco (1998), realizada en el marco de la Conferencia Mundial sobre Educación Superior, se afirma que la educación superior debe reforzar sus funciones de servicio a la sociedad, en lo relacionado con la erradicación de la pobreza, el hambre, el analfabetismo, la violencia, la intolerancia, el deterioro del medio ambiente, mediante esfuerzos interdisciplinarios para analizar los diferentes problemas. Sostiene también que los vínculos con el mundo del trabajo deben reforzarse, realizando intercambios de diversos tipos entre la academia y el mundo laboral.

Afirma la mencionada declaración que la pertinencia de la educación superior debe evaluarse en términos de la adecuación entre lo que la sociedad espera de las instituciones y lo que estas hacen. Para ello se debe propender por alcanzar una mejor articulación con los problemas de la sociedad y del mundo del trabajo; los propósitos de la educación superior deben tener como fin último las necesidades sociales, del respeto a las culturas y la protección del medio ambiente.

\footnotetext{
Dr. En Medio Ambiente y Desarrollo Sostenible, Ingeniero Industrial, Docente Asociado DAISIFII-UNMSM. E-mail: otinocog@gmail.com

** Ing. Industrial, Docente Asociado DAPGI-FII-UNMSM. E-mail: ismael.vizarreta@gmail.com
} 
Peralta (2010) en el marco del IV Congreso de Extensión Universitaria (Argentina) precisa acertadamente que el reconocimiento de la Universidad como institución social implica "aceptar que las definiciones institucionales se construyen en contextos históricos y por lo tanto, entender que la relación Universidad - Estado y Universidad - Sociedad estará marcada por los modelos de Estado y de desarrollo y las concepciones teóricas e ideológicas predominantes en cada contextos histórico".

En tal sentido, Tünnermann (2000), señala que la incorporación de la Extensión Universitaria y de la Difusión Cultural entre las tareas de la Universidad latinoamericana, forma parte de un proceso histórico encaminado a lograr una mayor apertura y democratización de la Universidad y una más amplia proyección social de su labor.

Resaltando el papel fundamental de la Reforma Universitaria de Córdoba, en el sentido de diferenciación histórica con las universidades occidentales, Castro Martinez (2009), citada por Gonzales (2013), señala que, como resultado de este movimiento, la universidad latinoamericana se autodefinió como una entidad de democratización y de reforma social, guiada por las actividades de enseñanza, investigación y "extensión" (esta última entendida como la colaboración directa con los sectores de la población menos favorecidos, a través de la difusión cultural y de la asistencia técnica).

Añade Castro Martinez, acertadamente, que mientras que en el Norte la "tercera misión" impulsa a las universidades a participar de forma directa en el desarrollo económico de su región, a través de una vinculación más estrecha con el sector productivo, en América Latina la adopción de la "extensión" como actividad académica, llevó a las universidades a participar más activamente en el desarrollo social de los pueblos, cubriendo, no pocas veces, los vacíos dejados por un Estado deficiente.

Mendez (2011) sostiene que el rol que le corresponde desempeñar a la extensión universitaria en el contexto de la sociedad contemporánea es el de la responsabilidad social; en tal sentido, propone que se incluya en los planes de estudio una asignatura denominada Responsabilidad Social, la misma que deberá incluir una actividad de Proyección Social que se relacione con asignaturas de cada especialidad.

Aponte (2007) señala que el ejercicio de la función de extensión universitaria debe realizarse a partir de la identidad misma de ella, en relación con las características y finalidad de cada especialidad. En el aspecto de gestión de la extensión universitaria propone la determinación de indicadores, enmarcados en los procesos de autoevaluación y acreditación universitaria.

Rama (2008) señala que la extensión universitaria es un sistema de prestación de servicios para los estudiantes y la comunidad; agrega que la tradicional concepción de la extensión universitaria se ha ido diluyendo, asociada a la democratización de las sociedades y a nuevas formas de expresión de la extensión, que incorporan la innovación, la inclusión social o la internacionalización.

Giménez (2000) señala que la Extensión Universitaria alberga y alimenta una finalidad y una fuerza centrífuga. En este sentido, pretendemos que la Universidad debe sentirse solidariamente implicada en todas las actividades humanas en las que pueda poner su contribución, que se puede concretar, en una doble dirección: hacia su propio alumnado y hacia su entorno exterior más próximo.

Tünnermann (2000), respecto a la extensión universitaria, señala que se debe concebir la función como una actividad estratégica de las instituciones de educación superior, en tanto que favorece la creatividad, la innovación y el contacto con el entorno, permitiendo la generación de respuestas oportunas y flexibles, evitando la obsolescencia.

Para González \& González (2013) la extensión universitaria es el proceso que tiene como propósito promover cultura en la comunidad intra y extrauniversitaria como parte de la contribución universitaria al desarrollo cultural.

González \& González (2013), en un análisis de la evolución y principales tendencias internacionales sobre la extensión universitaria en universidades latinoamericanas, resumen tres grandes modelos:

Modelo tradicional: en el que la universidad se concibe como fuente de conocimiento y saberes, y establece una relación de saber institucionalizado con quien no lo posee.

Modelo economicista: la universidad se concibe como una empresa más que interactúa en el mercado. Adquiere el rol de soporte científico y técnico del sector productivo, y el saber se organiza en función de la rentabilidad económica

Modelo de desarrollo integral: la universidad maneja el concepto de democratización del saber y asume la función social de contribuir a mejorar 
la calidad de vida de la sociedad. Apunta a la transformación social y económica de los pueblos.

Añaden Gonzales \& Gonzales (2013) que, teniendo en cuenta que la universidad es una institución cuyo referente es la sociedad y no el mercado, ella debe abrirse al medio social, desde los valores comunes a los miembros de la comunidad académica y científica.

Garcia (2010), en un balance crítico de la extensión universitaria en la Universidad de Cuyo, resume las principales limitaciones de esta función:

- Carencia de atributos esenciales

- Falta de integración en una propuesta global

- Falta de financiamiento permanente

- Falta de intervención en terreno

En una nueva perspectiva, Garcia da cuenta de los aspectos que se vienen implementando en esta universidad, con el nombre de prácticas sociales educativas, el mismo que aprobado por el Consejo Superior de la UBA (Resol. CS No 0520/2010). La propuesta implica la reforma curricular en todas las carreras de la Universidad, incluyendo 50 horas de tarea social obligatoria, requisito indispensable para realizar el trámite de título.

En la misma perspectiva, Macchiarola (2010) da cuenta de la implementación, en la Universidad de Cuyo, de la incorporación de prácticas comunitarias en la currícula, apuntando a la formación crítica y ciudadana del estudio y reafirmando el compromiso social de la universidad.

En el marco de las recientes experiencias y reflexiones sobre el rol de la extensión universitaria en Latinoamérica, Ramírez (2012) resume la perspectiva del CID. La Extensión Universitaria hace parte de los procesos misionales que posibilitan el cumplimiento de la Función Social de las instituciones de educación superior y tiene como propósito el desarrollo de procesos de interacción e integración con los agentes sociales y las demás funciones misionales.

Añade Ramírez que la extensión universitaria es una práctica social que aporta a las transformaciones sociales y al desarrollo de los entornos locales y regionales, pero desde su misión.

Concluye Ramírez (2012) que el reconocimiento de la Extensión como un proceso de interacción e integración, conlleva entre otras cosas a señalar que:

- La Extensión expresa la Responsabilidad Social de las instituciones educativas y la dimensión ética de sus comunidades académicas, relacionadas con su contribución a la comprensión y a la solución de los problemas del país en el ámbito nacional, regional y local.

- La Extensión permite establecer un diálogo permanente, respetuoso, riguroso y crítico, entre los saberes especializados de la academia (científicos, tecnológicos y artísticos) y los saberes y experiencias sociales, posibilitando una integración activa entre las IES y las instituciones sociales.

En la experiencia de la UNMSM y en el ámbito de la acreditación universitaria que establece el CONEAU, el estándar 56 establece claramente las pautas para evaluar las actividades de extensión universitaria, a través del seguimiento de los proyectos de extensión universitaria hasta su ejecución.

La base legal correspondiente respecto a extensión universitaria e investigación, para el caso sanmarquino, es:

- Ley Universitaria № 23733

- Estatuto de la UNMSM

- Reglamento General de la UNMSM

- Creación del Vicerrectorado de Investigación (RR N 03316-R-05 del 30/06/2005)

- Funciones de los organismos del VRI: Consejo Superior de Investigaciones (CSI), Consejo de Transferencia e Innovación, Consejo de Gestión de la Investigación (CGI)

- Manual de procedimientos de la Facultad de Ingeniería Industrial

En el ámbito de la investigación en la UNMSM; el Consejo Superior de Investigación (CSI) tiene como función fomentar, promover, registrar, evaluar y facilitar el desarrollo de las actividades de investigación en la UNMSM, brindando las facilidades de financiamiento de proyectos de investigación y en cuyo reglamento se establece la obligatoriedad de participación de docentes de pre grado y posgrado, como colaboradores.

Por su parte, el Consejo de Transferencia e Innovación (CTI) tiene entre sus funciones la de promover y gestionar las relaciones entre la universidad y la sociedad, representada por empresas, comunidades, instituciones científicas y culturales, públicas y privadas, y otras, en el área de ciencia y tecnología, específicamente a través de contratos de investigación, prestación de servicios y asesorías. 
EICGI aborda, a través de la Oficina de Planeamiento y Desarrollo, entre otras funciones, Esta Oficina está encargada de promover el desarrollo de actividades de investigación a nivel de estudiantes de pregrado y postgrado, orientadas a Desarrollar planes y programas de investigación asociados con sectores que generen ventajas competitivas para la UNMSM

Estas pautas generales que emanan del Vicerrectorado de Investigación de la UNMSM constituyen las bases para propiciar una estrecha relación entre las tareas de formación profesional, extensión universitaria e investigación en cada una de las Escuelas Académico profesionales de la facultad de Ingeniería Industrial.

Teniendo como norte la extensión universitaria, materia de esta investigación, es necesario establecer, y concordando con Vento (2012), se propone centrar en la actividad de extensión universitaria una estrategia que permita insertar los procesos de docencia, investigación y extensión, de modo tal que se contribuya a una formación profesional integral, vinculada a la realidad, que aporte a la comunidad y se nutra de ella para desarrollar las tareas principales de la universidad.

\section{LINEAMIENTOS PARA LAS FUNCIONES DE EXTENSIÓN UNIVERSITARIA E INVESTIGACIÓN EN LA FII}

Se formulan en el marco de una concepción integral de la extensión universitaria, como elemento integrador de las funciones básicas de formación profesional e investigación, y en coordinación con la propuesta de Vizarreta (2013) respecto a nuevas pautas para la extensión universitaria y proyección social en la facultad de Ingeniería Industrial de la UNMSM:

En los proyectos de Extensión Universitaria y Proyección Social de la FIl participarán obligatoriamente los alumnos de los ciclos 7 al 10, integrando grupos de alumnos por proyecto (número de integrantes por definir) y por cada etapa del proyecto y un docente Tutor, quien los guiará por cada proyecto y por cada una de las etapas siguientes:

1.- Etapa de elaboración del proyecto

2.- Etapa de desarrollo del proyecto

3.- Etapa de puesta en marcha del proyecto

4.- Etapa de seguimiento y mejora de los procesos del proyecto
La participación de los alumnos en estos proyectos deberá formar parte de la curricula obligatoria de la Escuela de Ing. Industrial, Escuela de Textil y Confecciones y otras que surjan en el futuro y dicha participación será un requisito obligatorio que debe reunir el alumno para declararlo egresado.

La participación de los docentes tutores, debe considerarse como horas lectivas en horarios establecidos, de la misma manera serán considerados los docentes que conforman el Comité Evaluador del CEUPS.

Cada etapa se realizará durante un período académico, según cronograma de fechas establecidas por el Comité Evaluador del CEUPS.

En la SEMANA 15 del ciclo los alumnos y docente tutor presentarán el informe de haber culminado la etapa que será evaluada por el Comité Evaluador del CEUPS.

El comité Evaluador del CEUPS determinará y/o coordinará la localidad donde debe desarrollarse el proyecto.

El comité Evaluador del CEUPS dará la conformidad de la culminación de la etapa, extendiéndole el certificado (previo pago, costo a definir) de haber participado en proyectos de Extensión Universitaria y Proyección Social, lo que le permitirá al alumno cumplir con un requisito para ser declarado egresado.

Al Docente Tutor se la dará una constancia por haber culminado la etapa, siempre y cuando cuente con la aceptada del Comité Evaluador del CEUPS, esta constancia le permitirá al docente acreditar su carga académica en proyectos de extensión Universitaria y Proyección Social FII

Previamente un Comité Evaluador del CEUPS integrado por docentes (CEUPS) evaluará los proyectos que presentan los alumnos, a fin de determinar la viabilidad del proyecto.

El Comité Evaluador del CEUPS asignará a los docentes tutores que guiarán a los alumnos en las diferentes etapas del proyecto.

El Comité Evaluador del CEUPS, evaluará los avances que presentas los grupos de trabajo integrados por los alumnos y la participación del docente tutor en las diferentes etapas del proyecto. 
El Comité Evaluador del CEUPS establecerá el cronograma de las fechas en que se deben presentar los proyectos, avances de las diferentes etapas, entrega del informe final de la etapa.

Para los casos de grupos que no cumplan con el cronograma de los controles establecidos no obtendrán sus certificados respectivos, tanto alumnos como docentes tutores.

EI CEUPS coordinará con el Instituto de Investigación de Ingeniería Industrial y las Escuelas de Ingeniería Industrial y la Textiles y Confecciones, las acciones y normativas para implementar la propuesta.

EI CEUPS coordinará con el Instituto de Investigación de Ingeniería Industrial y las instancias operativas del Vicerrectorado de Investigación, las facilidades y el financiamiento de los proyectos de Extensión Universitaria y Proyección Social.

Los términos de esta propuesta, que se asumen en esta investigación, permitirán mejorar la interacción Universidad - Sociedad, darle formalidad y rigurosidad a las tareas de extensión universitaria (presencia curricular) y puede ser el punto de partida para integrar investigación, extensión universitaria y formación profesional.

El conjunto de indicadores necesarios para esta propuesta incluye:

- Número de proyectos de Extensión Universitaria y Proyección Social por semestre, registrados en el CEUPS de la FII.

- Número de estudiantes asignados a los proyectos de Extensión Universitaria y Proyección Social por semestre, registrados en el CEUPS de la FII.

- Número de estudiantes asignados a los proyectos de Extensión Universitaria y Proyección Social por semestre, registrados en el CEUPS de la FII.

- Número de investigaciones derivadas de los proyectos de Extensión Universitaria y Proyección Social por semestre, registrados en el CEUPS de la FII.

- Número de tesis de investigación de pre y posgrado derivadas de los proyectos de Extensión Universitaria y Proyección Social por semestre, registrados en el CEUPS de la FII.

- Número de artículos de investigación publicados, derivadas de los proyectos de Extensión Universitaria y Proyección Social

\section{METODOLOGÍA Y TÉCNICAS DE INVESTIGA- CIÓN UTILIZADAS}

La investigación es de tipo aplicada, de carácter descriptivo, la misma que ha partido de un proceso de recopilación de experiencias y reflexiones sobre la extensión universitaria en Latinoamérica y la aproximación empírica, a través de encuestas, sobre la percepción de esta función en docentes y estudiantes de la facultad de Ingeniería Industrial.

\section{Recolección e interpretación de datos.}

El trabajo de campo se orientó a conocer la percepción de los estudiantes de la facultad de Ingeniería Industrial acerca de la función de extensión universitaria. Se consideró una muestra representativa y aleatoria de estudiantes de la facultad de Ingeniería Industrial, cuya composición fue la siguiente:

Cuadro 1. Muestra de alumnos según Escuela.

\begin{tabular}{|c|c|c|c|}
\hline \multicolumn{1}{|c|}{} & Frecuencia & Porcentaje \\
\hline \multirow{4}{*}{ Válidos } & Industrial & 88 & 87,1 \\
\cline { 2 - 4 } & $\begin{array}{c}\text { Textiles y } \\
\text { Confecciones }\end{array}$ & 13 & 12,9 \\
\cline { 2 - 4 } & Total & 101 & 100,0 \\
\hline
\end{tabular}

Fuente: Elaboración propia.

La distribución de la muestra por año de ingreso se muestra a continuación:

Cuadro 2. Muestra de alumnos según año de ingreso.

\begin{tabular}{|c|c|c|c|}
\hline \multicolumn{2}{|c|}{ Año ingreso } & Frecuencia & Porcentaje \\
\hline \multirow{4}{*}{ Válidos } & 2013 & 10 & 9.9 \\
\cline { 2 - 4 } & 2012 & 28 & 27.7 \\
\cline { 2 - 4 } & 2011 & 21 & 20.8 \\
\cline { 2 - 4 } & 2010 & 24 & 23.8 \\
\cline { 2 - 4 } & 2009 & 18 & 17.8 \\
\cline { 2 - 4 } & Total & 101 & 100.0 \\
\hline
\end{tabular}

Fuente: Elaboración propia. 


\section{Gráfico 1.}

Según el estatuto de la UNMSM, extensión universitaria y proyección social son funciones

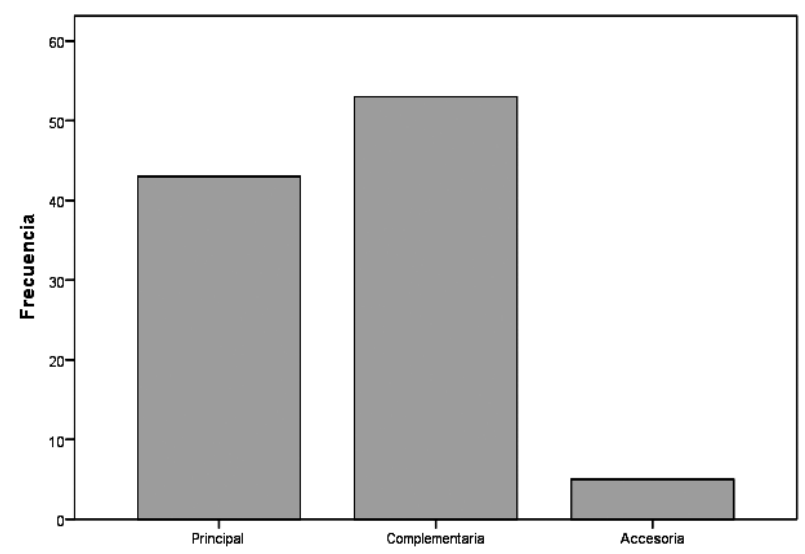

Fuente: Elaboración propia.

En el Gráfico 1 se muestran las respuestas de la muestra de estudiantes sobre el papel que le corresponde a la extensión universitaria, según el Estatuto UNMSM. La mayor proporción de estudiantes considera, erróneamente, que esta función es complementaria.

En el gráfico 2 se ilustra el nivel de conocimiento que tienen los estudiantes sobre las actividades de extensión universitaria o proyección social en la facultad de Ingeniería Industrial.

\section{Gráfico 2.}

¿Conoce Usted alguna actividad de proyección social realizada por el CEUPS-FII?

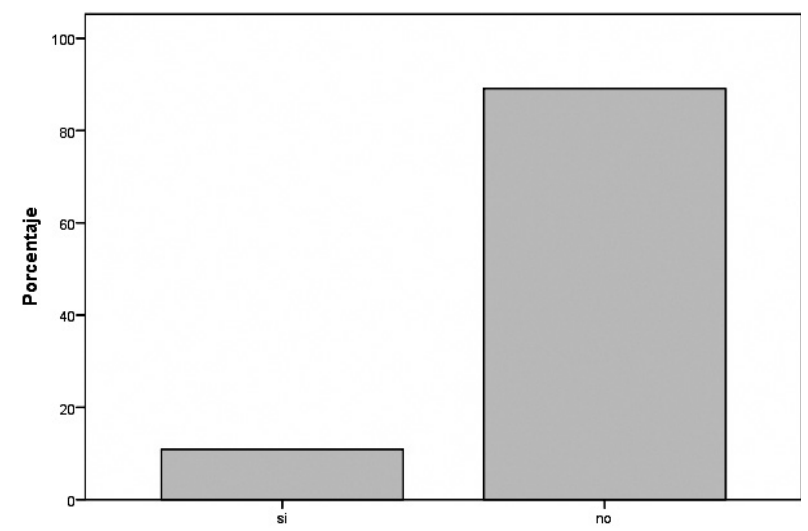

Fuente: Elaboración propia.
Una proporción mayoritaria de la muestra no conoce actividad de proyección social realizada por el CEUPS FII.

En igual sentido se puede interpretar el gráfico 3, en donde se pregunta si el estudiante ha participado en alguna actividad de proyección social en la FII UNMSM.

\section{Gráfico 3}

¿Ha participado Usted de algún proyecto de extensión universitaria y/o proyección social realizada por el CEUPS-FII?

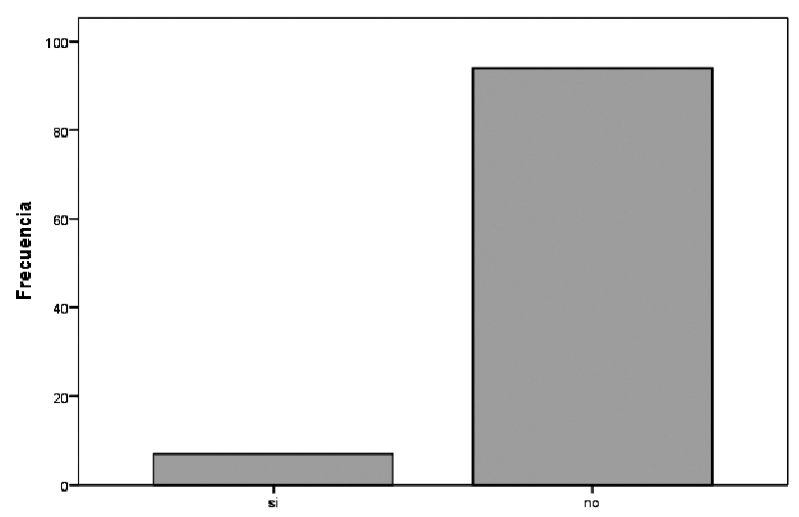

Fuente: Elaboración propia.

\section{CONCLUSIONES Y RECOMENDACIONES}

1. Es necesario asumir una concepción integral de extensión universitaria, que aproxime a la universidad a la integración e interacción con la comunidad de la que forma parte.

2. Las universidades latinoamericanas, dado el desarrollo de las economías en que se hallan insertas, tienen todavía el rol de aproximación social con la sociedad, siendo fundamental esta aproximación tanto para llevar conocimiento y cultura, como para recoger las experiencias que en ella se dan.

3. En el caso de la facultad de Ingeniería Industrial se proponen lineamientos para una nueva ejecutoria de esta importante función, teniendo en cuenta, además, que existe un marcado divorcio del estamento estudiantil de las actividades que le corresponden a la facultad en extensión universitaria y proyección social.

4. Se recomienda, a las autoridades de la facultad, consolidar la propuesta formulada en este trabajo y dar lugar, formalmente, de una visión integral 
de la extensión universitaria y su relación con las tareas de investigación y formación profesional.

\section{REFERENCIAS BIBLIOGRÁFICAS}

[1] Aponte, Claudia (2007). Propuesta de indicadores de evaluación de la función de proyección social/ extensión universitaria/ interacción en la educación superior .

[2] Castro, E. y J. Vega (2009). "Las relaciones universidad-entorno socioeconómico en el Espacio Iberoamericano del Conocimiento". Revista CTS, Volumen 4 (N. $\left.{ }^{\circ} 12\right)$, pp. 8, 9 y 10.

[3] Garcia, Oscar (2010). La extensión universitaria y su impacto curricular, prácticas sociales educativas. IV Congreso Nacional de Extensión Universitaria. Mendoza, Argentina.

[4] Giménez,Juan(2000).LaExtensiónUniversitaria en España. V Congreso Iberoamericano de Extensión. México, Universidad Michoacana de San Nicolás de Hidalgo.

[5] Gonzales y Gonzales (2013). ¿Extensión universitaria, proyección social o tercera misión? una reflexión necesaria. Revista Congreso Universidad. Vol. II, N. ${ }^{\circ} 2,2013$.

[6] Macchiarola, Viviana (2010). Incorporación de prácticas socio comunitarias en el currículo de la UNRC, Argentina. IV Congreso Nacional de Extensión Universitaria. Mendoza, Argentina.

[7] Mendez, Maria (2011). Experiencias de implementación de la extensión universitaria y proyección. Seminario Taller UNMSM.
[8] Peralta, Maria (2010). Compromiso social y calidad educativa: desafíos de la extensión universitaria. IV Congreso Nacional de Extensión Universitaria. Mendoza, Argentina.

[9] Ramirez, Carlos (2012). Algunas Consideraciones Extensión Universitaria en Colombia - Red Nacional de Extensión Universitaria. Segundo Encuentro Anual del Proyecto CID, Conocimiento, Inclusión, Desarrollo. Colombia.

[10]Tünnermann, Carlos (2000). El nuevo concepto de la extensión universitaria. Universidad Michoacana de San Nicolás de Hidalgo Morelia, Michoacán, México.

[11]Vento Tielves R, Pimentel Rivero I, Villarreal Domínguez J y RJ Valdés Corrales (2012). El papel de la Extensión Universitaria en la formación del profesional de la carrera de Agronomía de Montaña de San Andrés. Disponible en: http://www.fao.org/forestry/93400a3195715c9d8a75b88903b0def7152f.pdf. Último acceso: Diciembre 2013.

[12]Villanueva, E. (2008). Reformas de la educación superior: 25 propuestas para la educación superior en América Latina y el Caribe. En: IESALC UNESCO: Conferencia Regional de Educación Superior 2008. Venezuela.

[13]Vizarreta, Roberto (2013). Pautas para implementar la extensión universitaria y proyección social. UNMSM. Inédito. 